\title{
RESEARCH
}

\section{THE OUTCOMES OF USING FIXED-DOSE NOMOGRAM-GUIDED UNFRACTIONATED HEPARIN THERAPY IN ELDERLY PATIENTS IN COMPARISON WITH YOUNGER PATIENTS}

Turkish Journal of Geriatrics

DOI: 10.31086/tigeri.2020.167

2020; 23(3): 308-316

- Aynur ACIBUCA ${ }^{1}$ (D)

- İbrahim Haldun MÜDERRiSOĞLU²

CORRESPONDANCE

Aynur ACIBUCA

Baskent University School of Medicine,

Dr Turgut Noyan Application and Research

Center, Cardiology, Adana, Turkey

Phone: +903223272727

e-mail: aynuracibuca85@gmail.com

Received: Jun 20, 2020

Accepted: Aug 08, 2020

Baskent University School of Medicine,

Dr Turgut Noyan Application and Research

Center, Cardiology, Adana, Turkey

Baskent University School of Medicine,

Cardiology, Ankara, Turkey

\section{Abstract}

Introduction: In elderly patients, the dosing of unfractionated heparin is difficult because of changing metabolism, which affects the determination of a therapeutic level. The aim of this study was to evaluate the effects of unfractionated heparin therapy in an older population and to determine whether there was any difference from a younger population in terms of the activated partial thromboplastin time results and complication rates when using a standard nomogram.

Materials and Methods: A total of 120 patients aged <80 years (Group 1) and 120 patients aged $\geq 80$ years (Group 2 ), all of whom had been given unfractionated heparin therapy for selected indications in the coronary care unit, were randomly selected and retrospectively enrolled in the study. Infusion time up to a maximum of 48 hours was included, and activated partial thromboplastin time results in that period were categorised as subtherapeutic, therapeutic, or supratherapeutic. The rates of the patients' subtherapeutic, therapeutic, and supratherapeutic results were compared between groups.

Results: The peak activated partial thromboplastin time level was higher in patients aged $\geq 80$ years (58.85 [51.28] vs 76.50 [57.45], $p<0.001)$. The supratherapeutic activated partial thromboplastin time percentage $(0[0.25]$ vs $0.20[0.43], p<0.001)$ and numbers of first activated partial thromboplastin time in the supratherapeutic range ( $10 \%$ vs $24.2 \%, p=0.004$ ) were significantly higher in the older group.

Conclusion: Over-anticoagulation may be a problem in the elderly population when using standard nomograms. It was concluded that improvements should be made for a nomogram specific to an older population.

Keywords: Aged; Partial thromboplastin time; Heparin; Hemorrhage 


\section{INTRODUCTION}

The decision to administer anticoagulation is difficult in the elderly population due to the higher risk of haemorrhage and susceptibility to thrombosis. However, there are no specific recommendations about the use of anticoagulant medications for patients of advanced age.

Unfractionated heparin (UFH) is a parenteral anticoagulant that has been used for many years for different indications. The pharmacokinetics of UFH can vary because of interactions with various plasma proteins (1). Therefore, closely monitoring UFH's effects to reach optimal anticoagulation is the mainstay of heparin therapy. For this monitoring, the activated partial thromboplastin time (aPTT), which targets reaching and maintaining a therapeutic range, is commonly used. However, the unpredictable pharmacokinetics of UFH also affect the time taken to achieve a therapeutic level.

The management of UFH administration is challenging, especially in the elderly. Age-related decreased renal function in elderly patients can result in prolonged half-life and increased blood levels of anticoagulants (2). Although the mechanism is not fully understood, older patients are more sensitive to anticoagulants because of pharmacodynamic changes (3). This may also be because of binding to plasma proteins other than antithrombin, which causes alterations in the blood level (4).

Based on the aforementioned data, it was hypothesised that routine treatment schemes and doses of heparin, which are in general use, may lead to over-anticoagulation in the elderly. World Health Organization has declared recently the elderly age group as 80 years and older. Also, there is no previous clinical study in the literature investigating the variable effect of UFH in the above mentioned oldest age group. Therefore, the aim of this study was to compare the results of using the same nomogram for patients aged $<80$ years and patients aged $\geq 80$ years.

\section{MATERIALS AND METHOD}

A retrospective examination was made of the records of coronary care unit patients who had been treated with UFH according to the same nomogram between January 2012 and March 2020. Selected indications for anticoagulation included acute coronary syndromes, atrial fibrillation, mechanical heart valve, and intracardiac thrombus. Patients with pulmonary embolism or deep venous thrombosis were not enrolled in the study due to the use of a different nomogram. Patients with subcutaneous UFH administration, heparin infusion duration of less than 24 hours, or age of $<18$ years were excluded. A total of 120 patients aged $\geq 80$ years (Group 2) were randomly selected, and 120 patients aged $<80$ years (Group 1 ) were also selected randomly for comparison.

All 240 patients had been given a bolus UFH dose of 5000 units intravenously followed by an intravenous infusion of 1000 units/h. The aPTT levels were measured every 6 or 12 hours, and the infusion dose was managed according to the nomogram in current use in the study hospital (Table 1). The therapeutic range was defined as aPTT within 50-70 seconds.

The aPTT measurements were performed with Dade Actin FS (Siemens Healthcare Diagnostics, Marburg, Germany), which can detect aPTT values between 22.1 and 180 seconds. The test results were classified as therapeutic (50-70 seconds), subtherapeutic ( $<50$ seconds), or supratherapeutic (>70 seconds). The proportions of therapeutic (the patient's therapeutic aPTT results / the patient's total number of aPTT measurements), subtherapeutic, and supratherapeutic results were identified for each patient. Then, all the subtherapeutic aPTT ratios, calculated for each patient separately, were analysed to reach the mean and median values of subtherapeutic aPTT proportion distribution in 120 patients. The same calculations were also performed to display the distribution of 
Table 1. Nomogram of intravenous unfractioned heparin that is used in our hospital.

\begin{tabular}{|c|c|c|c|c|}
\hline aPTT(seconds) & Bolus, units & $\begin{array}{l}\text { Discontinue infusion, } \\
\text { minutes }\end{array}$ & $\begin{array}{l}\text { Change rate of } \\
\text { infusion (mL/hour)* }\end{array}$ & $\begin{array}{l}\text { Time of next } \\
\text { measurement, hours }\end{array}$ \\
\hline$<40$ & 3000 & 0 & +2 & 6 \\
\hline $40-49$ & 0 & 0 & +1 & 6 \\
\hline $50-70$ & 0 & 0 & 0 & next day \\
\hline $71-85$ & 0 & 0 & -1 & next day \\
\hline $86-100$ & 0 & 30 & -2 & 6 \\
\hline $101-150$ & 0 & 60 & -3 & 6 \\
\hline$>150$ & 0 & 60 & -6 & 6 \\
\hline
\end{tabular}

*1 ml fluid containig 50 IU heparin sodium aPTT: activated partial thromboplastin time.

therapeutic and supratherapeutic aPTT ratios in each group. The first sample measurement and peak value of aPTT for each patient were evaluated. The time until the achievement of therapeutic value was recorded and was categorized as $<12$ hours, 12-24 hours, or $>24$ hours. The heparin infusion time and APTT results were included up to the 48th hour. Unfractionated heparin infusion duration and aPTT measurements after 48 hours were excluded from the analysis.

Age, gender, comorbidities, and concomitant medications were evaluated. Data about new thrombosis and bleeding during UFH therapy was collected. Laboratory parameters on admission and during the haemorrhagic event were recorded. Approval for the study was granted by the Institutional Ethics Committee.

\section{Statistical analysis}

To determine the sample size required, the G-Power 3.1 program was used, and it was esti- mated that 92 patients would be required in each group to provide $80 \%$ power with a $95 \%$ confidence interval.

Statistical analyses were performed using SPSS version 26 software (SPSS Inc, Chicago, IL, USA). Categorical variables were reported as number (n) and percentage (\%). Continuous variables were presented as mean \pm standard deviation (SD) or median (interquartile range) values in the case of abnormal distribution. Comparisons of data between the two groups were made using Pearson chi-square or Fisher's exact test for categorical variables and with the paired t-test or Mann-Whitney $U$ test for continuous variables.

A value of $p<0.05$ was accepted as statistically significant for all analyses.

\section{RESULTS}

A total of 240 patients who had received UFH according to the same nomogram were evaluated. Group 1 consisted of 120 patients aged $<80$ years, 
and Group 2 consisted of 120 patients aged $\geq 80$ years. The age distribution of the study population is shown in Figure 1. The median age was 47 [15] years in Group 1 and 83 [5] years in Group 2. The baseline clinical characteristics of each group and laboratory parameters on admission are listed in Table 2.

The most common indications for UFH administration for Group 1 and Group 2 were acute coronary syndromes (67.5\% vs $83.3 \%)$, followed by atrial fibrillation ( $16.7 \%$ vs $14.2 \%)$, mechanical heart valve (15\% vs $1.7 \%)$, and intracardiac throm- bus $(0.8 \%$ vs $0.8 \%)$. Concomitant use of acetylsalicylic acid was more common in Group 2 (73.3\% vs $95.8 \%, \mathrm{p}<0.001)$, and warfarin use was more prevalent in Group 1 (17.5 vs 0\%, $p<0.001)$. The medications used at the same time as heparin and the comparison of the aPTT results between the two age groups are shown in Table 3. The peak aPTT level was higher in Group 2 (58.85 [51.28] vs 76.50 [57.45], $\mathrm{p}<0.001)$. The supratherapeutic aPTT ratio (0 [0.25] vs 0.20 [0.43], $\mathrm{p}<0.001$ ) and numbers of first aPTT in the supratherapeutic range $(10 \%$ vs $24.2 \%, p=0.004$ ) were significantly higher in Group

Table 2. Nomogram of intravenous unfractioned heparin that is used in our hospital.

\begin{tabular}{|c|c|c|c|}
\hline & Group $1(n=120)$ & Group 2(n=120) & $p$ value \\
\hline Male & $84(70)$ & $46(38.3)$ & $<0.001^{*}$ \\
\hline Hypertension & $43(35.8)$ & $79(65.8)$ & $<0.001^{\star}$ \\
\hline Diabetes mellitus & 25(20.8) & $41(34.2)$ & $0,021^{*}$ \\
\hline Chronic renal disease & $10(8.3)$ & $39(32.5)$ & $<0.001^{\star}$ \\
\hline Haemodialysis & $6(5)$ & $2(1.7)$ & 0,281 \\
\hline Heart failure & 18(15) & $41(34.2)$ & $0,001 *$ \\
\hline Creatinine, $\mathrm{mg} / \mathrm{dL}$ & $0.84[0.36]$ & $1.12[0.68]$ & $<0.001^{\star}$ \\
\hline Hematocrit, $\%$ & $40.96 \pm 6.53$ & $36.99 \pm 4.89$ & $<0.001^{\star}$ \\
\hline White blood cells, $10^{\wedge} 3 / \mu l$ & $11[5.24]$ & $8.85[4.57]$ & $<0.001$ \\
\hline Platelets, $10^{\wedge} 3 / \mu \mathrm{l}$ & $246.50[124]$ & $220.50[114]$ & $0,010^{*}$ \\
\hline C-reactive protein, $\mathrm{mg} / \mathrm{L}$ & 8.44 [22.03] & $13.25[37.20]$ & 0,058 \\
\hline
\end{tabular}

Categorical variables are presented as numbers and percentage $\mathrm{n}(\%)$. Continuous variables are presented as mean \pm standart deviation or median [interquartile range]

* $p<0.05$ accepted as statically significant 
Table 3. Medications given concomitantly with unfractionated heparin and the distribution of aPTT test results.

\begin{tabular}{|c|c|c|c|}
\hline & Group $1(n=120)$ & Group $2(n=120)$ & $P$ value \\
\hline Acetylsalicylic acid,n & $88(73.3)$ & $115(95.8)$ & $<0.001^{*}$ \\
\hline Clopidogrel ,n & $36(30)$ & $26(21.7)$ & 0,140 \\
\hline Prasugrel-tikagrelor, $\mathrm{n}$ & $4(3.3)$ & $0(0)$ & 0,122 \\
\hline GP Illb-IIla antagonists, $n$ & $15(12.5)$ & $4(3.3)$ & $0,009^{\star}$ \\
\hline Warfarin, $\mathrm{n}$ & $21(17.5)$ & $0(0)$ & $<0.001^{\star}$ \\
\hline Heparin infusion duration,h & $36.16 \pm 9.98$ & $35.45 \pm 9.93$ & 0,582 \\
\hline $\begin{array}{l}\text { Subtherapeutic aPTT } \\
\text { proportion }\end{array}$ & $0.67[0.60]$ & $0.46[0.47]$ & $<0.001^{\star}$ \\
\hline Therapeutic aPTT proportion & $0.15[0.33]$ & $0.33[0.36]$ & $<0.001^{*}$ \\
\hline $\begin{array}{l}\text { Supratherapeutic aPTT } \\
\text { proportion }\end{array}$ & $0[0.25]$ & $0.20[0.43]$ & $<0.001^{*}$ \\
\hline First time aPTT, s & $37.20[18.10]$ & 48.25 [31.40] & $<0.001^{*}$ \\
\hline $\begin{array}{l}\text { First aPTT in subtherapeutic } \\
\text { range, } n\end{array}$ & $91(75.8)$ & $63(52.5)$ & $<0.001^{*}$ \\
\hline $\begin{array}{l}\text { First aPTT in therapeutic } \\
\text { range,n }\end{array}$ & $17(14.2)$ & $28(23.3)$ & 0,069 \\
\hline $\begin{array}{l}\text { First aPTT in supratherapeutic } \\
\text { range, } n\end{array}$ & $12(10)$ & $29(24.2)$ & $0,004^{*}$ \\
\hline Peak level of aPTT, s & $58.85[51.28]$ & $76.50[57.45]$ & $<0.001^{*}$ \\
\hline
\end{tabular}

Categorical variables are presented as numbers and percentage $\mathrm{n}(\%)$. Continuous variables are presented as mean \pm standart deviation or median [interquartile range]. s:seconds h:hours

$p<0.05$ accepted as statically significant

2 , suggesting that the older patients were more susceptible to the heparin effects.

When patients who could not reach the therapeutic range during UFH infusion were excluded, the time taken to achieve a therapeutic aPTT was not statistically different for the remaining patients in both groups ( $p=0.561$ ). In Group 1, $49.1 \%$ of the patients, and in Group 2, 24.2\% of the patients, did not reach the therapeutic range during UFH therapy. Time durations to achieve therapeutic 
aPTT are compared in Table 4.

Heparin-induced thrombocytopenia was not observed in any patient. Bleeding during UFH administration was detected in 8 (6.7\%) patients in Group 1, only one of which was a major haemorrhage. Bleeding occurred in $3(2.5 \%)$ patients in Group 2, one of which was major. The two age groups were not significantly different in terms of bleeding incidence $(p=0.123)$. The aPTT values at the time of bleeding were subtherapeutic in 5 (45.4\%) patients, therapeutic in $2(18.2 \%)$ patients, and suprathera-peutic in $4(36.4 \%)$ patients. None of the patients experienced a thrombotic event during UFH administration.

\section{DISCUSSION}

In this study, the aPTT results under UFH therapy were compared between two different age groups, and the results showed that dosing according to the same heparin nomogram resulted in higher peak values and a higher proportion of supratherapeutic aPTT, even in the first sample, in the older age group.

Due to changes in metabolism, elderly patients are susceptible to drug complications. In addition to pharmacokinetic changes, the pharmacodynamics of UFH also alter with age as a result of changing body weight and composition. More- over, UFH binds to numerous plasma proteins other than antithrombin, which causes variations in distribution volume and, consequently, changing plasma levels (4). It has been previously reported that therapeutic aPTT levels can be achieved with lower doses of UFH in older patients (5). In the current study, all age groups were given the same doses of heparin according to a standard nomogram, and the older patients had higher aPTT values as expected.

The elderly are at high risk of bleeding and thrombosis. Evaluating the risk/benefit ratio of anticoagulation in this population is still challenging because patients with a higher risk of haemorrhage will benefit more from anticoagulation based on current risk scores. However, anticoagulant levels that are too low or too high bring about the threat of new thrombotic events and bleeding. Moreover, the age of the patient has been reported to be a major risk factor for haemorrhagic complications, even in therapeutic aPTT values (6). Campbell et al. (5) reported that the incidence of bleeding and major bleeding in patients aged $\geq 72$ years was higher compared with those aged $<72$ years ( $14.1 \%$ vs $7.1 \%$ for bleeding and $11.1 \%$ vs $3.1 \%$ for major bleeding). In contrast with those findings, bleeding was encountered more in the younger age group in the current study, although not at a

Table 4. Time duration to achieve therapeutic aPTT

\begin{tabular}{|l|l|l|}
\hline & Group $\mathbf{1}(\mathbf{n = 1 2 0 )}$ & Group 2 (n=120) \\
\hline Never & $59(49.1)$ & $29(24.2)$ \\
\hline$<12$ hours & $28(23.3)$ & $46(38.3)$ \\
\hline $12-24$ hours & $21(17.5)$ & $24(20)$ \\
\hline$<24$ hours & $12(10)$ & $21(17.5)$ \\
\hline
\end{tabular}

The data are presented as numbers and percentages. 
Figure 1. Age distribution of the study population.

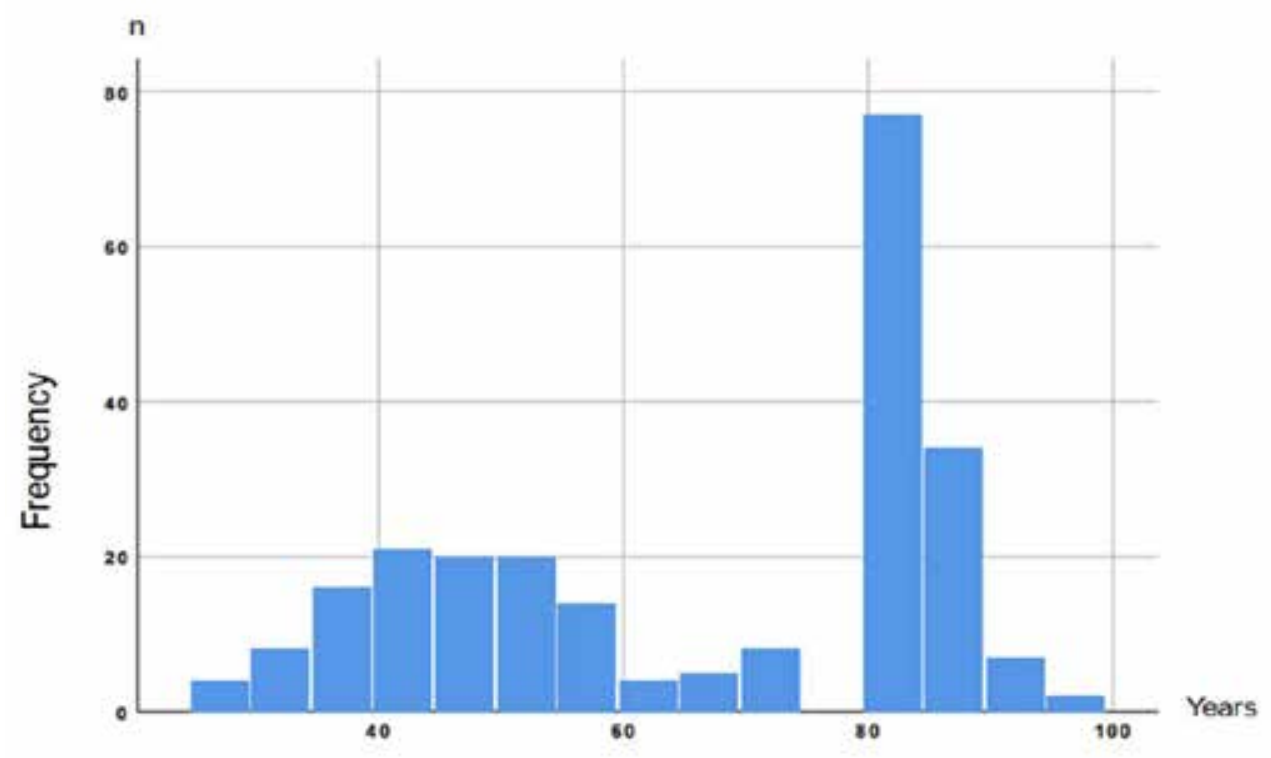

\section{Patient age}

statistically significant level. This result may have been due to the significantly higher prevalence of concomitant treatment with clopidogrel, warfarin, or GP Ilb-IIla antagonists in Group 1.

Whereas some researchers have reported that the prevalence of haemorrhage was associated with aPTT results, others could not reveal this association (7-10). The current study's findings also support this conflict because most of the bleeding occurred at therapeutic or subtherapeutic levels. Haemorrhagic complications occurred in only 4 $(36.4 \%)$ of the bleeding events when aPTT was supratherapeutic.

Although thrombotic events were expected because of the high proportion of subtherapeutic aPTT values (0.67 [0.60] in Group 1 vs 0.46 [0.47] in Group 2, $p<0.001)$, no new thromboembolic events occurred in either age group. This result may be attributable to the relatively short observation time in the hospital. There was no screening for any post-discharge embolic event that might have occurred as a result of thrombus formed previously during the subtherapeutic range. However, the high amount of subtherapeutic percentages may also have been due to the temporary interruption of anticoagulation before coronary angiography. As this was a retrospective study, it was not possible to be sure whether an aPTT measurement was performed at the time of terminating the heparin infusion.

The American College of Chest Physicians (ACCP) has recommended using a fixed-dose, as was used in this study, or a weight-based nomogram for UFH treatment, but there is no clarity as to which is superior (11). The use of a weight-adjusted heparin nomogram to reduce the risk of over-anticoagulation may be preferable, but in this case, the physicians must spare enough time for dose calculation to avoid medication errors.

The total dose and administration rate of UFH 
were not recorded in this study because the aim was to evaluate the percentage of aPTT results in subtherapeutic, therapeutic, and supratherapeutic ranges according to a standard nomogram.

\section{Limitations}

There are several limitations of the current study. First, because of the retrospective design, it was not certain whether each aPTT measurement was performed at the exact time. Second, the results are based on only the first 48 hours of the anticoagulation, and therefore, any delayed event of haemorrhage or thromboembolism may have been overlooked, as patients were not followed up after discharge. Finally, although the number

\section{REFERENCES}

1. Hylek EM, Regan S, Henault LE, et al. Challenges to the effective use of unfractionated heparin in the hospitalized management of acute thrombosis. Arch Intern Med 2003;163:621-7. (PMID:12622610)

2. Aymanns C, Keller F, Maus S, et al. Review on pharmacokinetics and pharmacodynamics and the aging kidney. Clin J Am Soc Nephrol 2010;5(2):314 27. (PMID:20056753)

3. Mangoni AA, Jackson SH. Age-related changes in pharmacokinetics and pharmacodynamics: basic principles and practical applications. $\mathrm{Br} \mathrm{J}$ Clin Pharmacol 2004;57(1):6-14. (PMID:14678335)

4. Hirsh J, Bauer KA, Donati MB, et al. Parenteral anticoagulants: American College of Chest Physicians Evidence-Based Clinical Practice Guidelines (8th Edition). Chest 2008;133(6):141-59. (PMID:18574264)

5. Campbell NR, Hull RD, Brant R, et al. Aging and heparin-related bleeding. Arch Intern Med 1996;156(8):857-60. (PMID: 8774204)

6. Schulman S, Beyth RJ, Kearon C, et al. Hemorrhagic complications of anticoagulant and thrombolytic treatment: American College of Chest Physicians Evidence-Based Clinical Practice Guidelines (8th Edition). Chest 2008;133(6 Suppl):257-98. (PMID:18574268)

7. Cossette B, Pelletier ME, Carrier N, et al. Evaluation of patients was sufficient to reach statistical significance, further studies with larger populations are needed to prove the existence of an association between patient age and aPTT results.

\section{CONCLUSION}

Using a standard heparin nomogram for all age groups can result in a high percentage of supratherapeutic aPTT results in the elderly. This finding supports the need for adopting a specific UFH nomogram for older patients. To find the optimal dosing scheme and frequency of aPTT measurements, further trials should be conducted in the older age group.

Conflict of interest: none declared.

of bleeding risk in patients exposed to therapeutic unfractionated or low-molecular-weight heparin: a cohort study in the context of a quality improvement initiative. Ann Pharmacother 2010;44(6):994-1002. (PMID:20442353)

8. Bauer SR, Ou NN, Dreesman BJ, et al. Effect of body mass index on bleeding frequency and acti-vated partial thromboplastin time in weight-based dosing of unfractionated heparin: a retrospective cohort study. Mayo Clin Proc 2009;84(12):1073-8. (PMID:19955244)

9. Thomas MP, Mahaffey KW, Chiswell K, et al. Activated partial thromboplastin time measurement is not associated with clinical outcomes in patients with high-risk non-ST-segment elevation acute coronary syndromes treated with unfractionated heparin. J Thromb Thrombolysis 2012;34(1):114-9. (PMID:22323092)

10. Floroff CK, Palm NM, Steinberg DH, et al. Higher maximum doses and infusion rates compared with standard unfractionated heparin therapy are associated with adequate anticoagulation without increased bleeding in both obese and non-obese patients with cardiovascular indications. Pharmacotherapy 2017;37(4):393-400. (PMID:28107569)

11. Holbrook A, Schulman S, Witt DM, et al. Evidencebased management of anticoagulant therapy: 
Antithrombotic Therapy and Prevention of Thrombosis, 9th ed: American College of Chest

Physicians Evidence-Based Clinical Practice

Guidelines. Chest 2012;141(2 Suppl):e152-84.

(PMID:22315259) 